\title{
CONTENIDO DE PLOMO, CADMIO Y ARSÉNICO EN TEJIDOS BIOLÓGICOS DE LA PALOMA COMÚN (Columba livia) PRESENTES EN UN ÁREA URBANA PREVIAMENTE CONTAMINADA CON RESIDUOS MINEROS
}

\author{
Content of lead, cadmium and arsenic in biological tissues of the feral pigeons (Columba livia) \\ present in an urban area previously contaminated with mining residues
}

\section{Pablo VALLADARES-FAUNDEZ ${ }^{1 *}$, Grimanesa CÁCERES TAPIA² y Jorge VALDÉS SAAVEDRA ${ }^{3}$}

${ }^{1}$ Departamento de Biología, Facultad de Ciencias, Universidad de Tarapacá, General Velásquez 1775 , Arica, Chile

${ }^{2}$ Departamento de Química, Facultad de Ciencias, Universidad de Tarapacá, General Velásquez 1775, Arica, Chile

${ }^{3}$ Laboratorio de Sedimentología y Paleoambiente (LASPAL), Instituto de Ciencias Naturales Alexander von Humboldt, Facultad de Ciencias del Mar y de Recursos Biológicos, Universidad de Antofagasta. Avenida Universidad de Antofagasta 02800, Antofagasta, Chile

*Autor para correspondencia: pvalladares@uta.cl

(Recibido: junio 2018; aceptado: agosto 2019)

Palabras clave: bioindicadores, metales pesados, Chile, contaminación

\section{RESUMEN}

El norte de Chile presenta numerosos casos de contaminación ambiental por metales pesados y otros químicos, particularmente debido al manejo inadecuado de desechos. En la ciudad de Arica, norte de Chile, se depositaron en la década de 1980 alrededor de 19 mil toneladas de desechos mineros provenientes de Europa, con altos contenidos de $\mathrm{Pb}$, As y Cd. Sus efectos sólo han sido evaluados en la población humana, pero hasta ahora se desconoce si esos elementos químicos fueron incorporados por otras especies que son parte de la comunidad biológica del radio urbano. En este estudio evaluamos la presencia y concentración de $\mathrm{Pb}$, As y $\mathrm{Cd}$ en palomas (Columba livia) que habitan en la ciudad de Arica, tanto en el radio urbano como en un valle aledaño (valle de Azapa). Los valores encontrados son altos en relación con otras aves de similares características ecológicas, por ejemplo respecto del $\mathrm{Pb}$ se encontraron en promedio $1.7 \mu \mathrm{g} / \mathrm{g}$ en tejido hepático y $10.84 \mu \mathrm{g} / \mathrm{g}$ en tejido óseo. En relación con el As, la concentración en hígado fue de $2.63 \mu \mathrm{g} / \mathrm{g}$ y en hueso de $2.13 \mu \mathrm{g} / \mathrm{g}$; finalmentela concentración de Cd en hígado fue de $11.7 \mu \mathrm{g} / \mathrm{g}$ y en hueso de $3.43 \mu \mathrm{g} / \mathrm{g}$. Esto indica que las palomas de la ciudad de Arica están altamente contaminadas por metales pesados y As, y podrían considerarse como una especie bioindicadora para futuras evaluaciones ambientales de la ciudad.

Key words: bioindicator, heavy metals, Chile, pollution

\begin{abstract}
The north of Chile presents numerous cases of environmental contamination by heavy metals and others chemical elements, particularly due to the inadequate handling of its waste. In the Arica city, northern Chile, in the decade of 1980, 19000 tons of mining
\end{abstract}


waste were deposited from Europe, with high contents of $\mathrm{Pb}, \mathrm{As}$ and $\mathrm{Cd}$. Its effects have only been evaluated in the human population, but until now it is unknown if those chemical elements were incorporated by other species that are part of the biological community of the urban radius. In this study we evaluated the presence and concentrations of $\mathrm{Pb}$, As and $\mathrm{Cd}$ in feral pigeons (Columba livia) inhabitants of Arica, both in the urban radius and in a nearby valley (valley of Azapa). The values found are higher in relation to other birds of similar ecological characteristics. For example, regarding $\mathrm{Pb}, 1.7 \mu \mathrm{g} / \mathrm{g}$ were found on average in liver tissue and $10.84 \mu \mathrm{g} / \mathrm{g}$ in bone tissue. In relation to As, concentrations were $2.63 \mu \mathrm{g} / \mathrm{g}$ in liver tissue and $2.13 \mu \mathrm{g} / \mathrm{g}$ in bone. Finally, concentrations of $\mathrm{Cd}$ in liver were $11.7 \mu \mathrm{g} / \mathrm{g}$ and $3.43 \mu \mathrm{g} / \mathrm{g}$ in bones. This indicates that the common pigeons from Arica are highly contaminated by heavy metals and arsenic and could be considered an environmental bioindicator species for future environmental assessment of the city.

\section{INTRODUCCIÓN}

Entre 1984 y 1989 se depositaron en la ciudad de Arica alrededor de 19 mil toneladas de residuos mineros con altos contenidos en $\mathrm{As}, \mathrm{Pb}$ y otros metales pesados. El origen de dichos acopios tiene relación con el ingreso de residuos industriales provenientes de Suecia para su procesamiento por parte de la empresa Procesadora de Metales Ltda. Junto con esto, la contaminación por $\mathrm{Pb}$ se acrecentó por el aumento de ferrocarriles y camiones bolivianos transportadores de desechos mineros, aumentando la exposición de la población a estos contaminantes (Montenegro 2009). Debido a ello, se han realizado numerosas mediciones de $\mathrm{Pb}$ y As en población humana, evidenciado niveles mayores a los referenciados por la Organización Mundial de la Salud (OMS), es decir sobre los $10 \mu \mathrm{g} / \mathrm{dL}$ (GORE 2009).

En 2009 comenzó a desarrollarse un plan de descontaminación en la ciudad, que contempla la reubicación de viviendas directamente aledañas a residuos, limpieza y aspiración de residuos en viviendas no aledañas, traslado de residuos mineros a zonas alejadas de la ciudad en contenedores herméticos, construcción de un galpón de acopio, pavimentación de suelos contaminados, evaluación de niveles de $\mathrm{Pb}$ y As en suelos de patios y en polvo sedimentable en colegios y liceos, remediación de suelos en líneas del ferrocarril, y evaluación de plombemia en la población expuesta y a alumnos de establecimientos educacionales aledaños a la zona de contaminación (GORE 2009). Sin embargo, a pesar de toda la información técnica levantada sobre este problema, nada se sabe de las consecuencias de esta contaminación en las comunidades biológicas, como por ejemplo en la fauna urbana.

Las aves han sido un grupo ampliamente utilizado en el biomonitoreo de áreas de interés ambiental (Pollak et al. 2017). Entre ellas, se han usado aves de eslabones tróficos superiores, como rapaces y necrófagas (buitres y jotes) (Dauwe et al. 2003, Hernández y Margalida 2009, Naccari et al. 2009, Valladares et al. 2013), pero también se han evaluado otras especies consumidoras primarias y omnívoras (Adout et al. 2007, Abbasi et al. 2015, Janaydeh et al. 2016).

Entre las especies adecuadas para desarrollar biomonitoreos ambientales, se encuentra la paloma común (Columba livia), especie muy abundante en los radios urbanos del mundo, con poblaciones de gran tamaño que no presentan problemas de conservación. En esta especie, que ha sido ampliamente utlizada como bioindicadora (Hoff y Antoniosi 2011) se ha evaluado la presencia de metales pesados en plumas (Hoff y Antoniosi 2011, Frantz et al. 2012, Lee et al. 2016) y otros tejidos internos (Nam et al. 2004, Nam y Lee 2006, Hoff y Antoniosi 2011, Cai y Calisi 2016).

En Chile, las palomas aún no han sido consideradas como bioindicadoras de contaminación, ya que hasta ahora solamente se han desarrollado en ellas estudios relacionados con parásitos y salud pública (Toro et al. 1999, González-Acuña et al. 2007). Por tal motivo, el objetivo de este trabajo es evaluar la presencia y concentraciones de $\mathrm{Pb}, \mathrm{Cd}$ y $\mathrm{Zn}$ en una población de palomas habitantes en el radio urbano de la ciudad de Arica, Chile, en hígado y hueso, y comparar estos resultados con especies de otras latitudes.

\section{MATERIALES Y MÉTODOS}

\section{Área de estudio y recolección}

Se recolectaron 26 especímenes de palomas en dos lugares del radio urbano de la ciudad de Arica, en el campus Velásquez de la Universidad de Tarapacá $\left(18^{\circ} 28^{\prime} 16^{\prime \prime} \mathrm{S}-70^{\circ} 18^{\prime} 41^{\prime \prime} \mathrm{O}\right)$ y en el kilómetro $1 \mathrm{del}$ 
valle de Azapa (18 31' 2" S-70 11' 31" O). Todos los ejemplares fueron recolectados con trampas Tomahawk en julio de 2017, de acuerdo con lo establecido en el permiso de caza Resolución Exenta $\mathrm{N}^{\circ}$ 1045/2017 del Servicio Agrícola y Ganadero de Chile, sacrificados con pentobarbital sódico según recomendaciones bioéticas (Close 2011) y procesados en el Laboratorio de Zoología Integrativa, Universidad de Tarapacá. Se analizaron tejidos hepáticos y óseos (fémur) para el análisis de tres elementos químicos, $\mathrm{Pb}$, As y $\mathrm{Cd}$. Los análisis químicos se realizaron en el Laboratorio de Sedimentología y Paleoambiente (LASPAL), Instituto de Ciencias Naturales Alexander von Humboldt, Universidad de Antofagasta.

\section{Pretratamiento de las muestras y análisis químico}

Todo el material plástico y de vidrio utilizado fue previamente tratado con soluciones de $\mathrm{HNO}_{3} 2 \mathrm{M}$ por $10 \mathrm{~h}, \mathrm{HCl} 2 \mathrm{M}$ por $10 \mathrm{~h}$, ácido etilendiaminotetraacético (EDTA) $0.01 \mathrm{M}$ por $10 \mathrm{~h}$ y finalmente enjuagado con abundante agua desionizada. Previo al lavado con agua desionizada (milliQ), el tejido blando húmedo fue homogenizado en mortero de ágata. Para la determinación de los elementos químicos analizados, entre 0.5 y $1 \mathrm{~g}$ de muestra húmeda (en triplicado) fueron puestas en un sistema de reflujo (es decir, un embudo de vidrio tapado con un vidrio reloj) y puestos en un vaso de precipitado de $125 \mathrm{~mL}$ para su disgregación con $10 \mathrm{~mL}$ de $\mathrm{HNO}_{3}$ Suprapur; luego fueron calentadas en una placa calefactora a $150^{\circ} \mathrm{C}$ por $2 \mathrm{~h}$. Posteriormente, la solución resultante fue filtrada $(0.45 \mu \mathrm{m})$ y aforada en un matraz volumétrico de $25 \mathrm{~mL}$ con agua desionizada. La cuantificación de metales fue determinada por espectrofotometría de absorción atómica (Shimadzu, AA 6300) y el As por generador de hidruros, con límites de detección (en $\mathrm{mg} / \mathrm{kg}$ ) de 0.27 para el $\mathrm{Pb}, 0.03$ para el Cd y 0.02 para el As. Las concentraciones se expresaron en $\mu \mathrm{g} / \mathrm{g}$ peso seco de tejido hepático y óseo, y el cálculo se aplicó según factor de dilución de la alícuota. Para el Pb se utilizó corrector de fondo. El procedimiento analítico fue revisado mediante el uso de material de referencia certificado DORM-3 (Dogfish) adquirido desde la National Research Council of Canada (NRC). Para el Pb, DORM-3 tuvo una concentración media de $0.373 \pm 0.04 \mu \mathrm{g} / \mathrm{g}$, con un valor de referencia de 0.39; en el caso del Cd, DORM-3 fue de $0.290 \pm 0.06$ $\mu \mathrm{g} / \mathrm{g}$, y valor de referencia de 0.29 , y finalmente el As se obtuvo a partir del patrón TORT-2, con una concentración media de $21.697 \pm 0.13 \mu \mathrm{g} / \mathrm{g}$ y valor de referencia de 21.6. En todos los metales analizados se observó gran precisión respecto al material de referencia (Castillo y Valdés 2011).

\section{Análisis estadístico}

Para comparar la magnitud de estos tres elementos químicos en las palomas del radio urbano de Arica se usó información bibliográfica de análisis realizados en otras latitudes. El análisis estadístico fue desarrollado en el programa SPSS 10.0 y consistió en la prueba no paramétrica U de Mann Whitney aplicada a muestras independientes para comparar las concentraciones de los tres elementos químicos entre tejidos y entre localidades de recolección, con un nivel de significancia de $\mathrm{p}<0.05$.

\section{RESULTADOS}

Considerando a todos los individuos $(\mathrm{n}=26)$, las concentraciones de $\mathrm{Pb}$ en hígado promediaron 1.7 $\mu \mathrm{g} / \mathrm{g}$, con un mínimo de $0.26 \mu \mathrm{g} / \mathrm{g}$ y un máximo de $6.41 \mu \mathrm{g} / \mathrm{g}$ (con desviación estándar $[\mathrm{DS}]=1.259$ ), mientras que en tejido óseo promediaron $10.84 \mu \mathrm{g} / \mathrm{g}$ y variaron de $6.83 \mu \mathrm{g} / \mathrm{g}$ a $37.88 \mu \mathrm{g} / \mathrm{g}(\mathrm{DS}=6.03)$. En el caso del $\mathrm{Cd}$ en hígado, el valor promedio fue de 11.69; el mínimo encontrado fue de $3.01 \mu \mathrm{g} / \mathrm{g}$ y el máximo de $42.63 \mu \mathrm{g} / \mathrm{g}(\mathrm{DS}=8.38)$. En tejido óseo el promedio fue de $3.43 \mu \mathrm{g} / \mathrm{g}$, con un valor mínimo de $0.38 \mu \mathrm{g} / \mathrm{g}$ y un máximo de $10.03 \mu \mathrm{g} / \mathrm{g}(\mathrm{DS}=$ 2.75). Finalmente, con relación al As, el promedio encontrado en tejido hepático fue de $2.63 \mu \mathrm{g} / \mathrm{g}$, con un valor mínimo de $0.52 \mu \mathrm{g} / \mathrm{g}$ y un valor máximo de $8.06 \mu \mathrm{g} / \mathrm{g}(\mathrm{DS}=1.59)$. En tejido óseo, el promedio fur de $2.13 \mu \mathrm{g} / \mathrm{g}$, con un mínimo de $1.07 \mu \mathrm{g} / \mathrm{g}$ y un máximo de $8.19 \mu \mathrm{g} / \mathrm{g}(\mathrm{DS}=1.35)$ (Cuadro I).

Al comparar las concentraciones entre tejidos, para un mismo elemento químico y por medio de la prueba U de Mann Whitney, se encontró que en el caso del As no hubo diferencias significativas entre tejido hepático y óseo $(Z=-1.501, p=0.133)$. En cuanto al $\mathrm{Pb}$, efectivamente hubo diferencias significativas entre los tejidos $(Z=-6.186, p=0.000)$, al igual que en el caso del Cd $(Z=-4.795, p=0.000)$. Finalmente, al comparar por este mismo método los dos sectores evaluados, no se observaron diferencias significativas en ninguno de los sitios analizados, es decir, el centro de la ciudad de Arica y el valle de Azapa, lo que podría indicar que las palomas se comportan como una sola gran población (As: $\mathrm{Z}=-1.464, \mathrm{p}=0.143, \mathrm{~Pb}: \mathrm{Z}=$ $-0.403, p=0.687, \mathrm{Cd}: \mathrm{Z}=-1.208, \mathrm{p}=0.227)$.

\section{DISCUSIÓN}

Las palomas (Columba livia) son aves que han sido permanentemente usadas como bioindicadoras 
CUADRO I. CONCENTRACIONES DE As, Pb y Cd* EN TEJIDOS DE PALOMA COMÚN (Columba livia) DE DOS LOCALIDADES DE LA CIUDAD DE ARICA, CHILE

\begin{tabular}{|c|c|c|c|c|c|c|c|}
\hline & & \multicolumn{2}{|c|}{ As } & \multicolumn{2}{|c|}{$\mathrm{Pb}$} & \multicolumn{2}{|c|}{$\mathrm{Cd}$} \\
\hline & & Hígado & Hueso & Hígado & Hueso & Hígado & Hueso \\
\hline \multirow{4}{*}{ Ciudad de Arica } & $\mathrm{MIN}$ & 0.5223 & 10.662 & 0.2591 & 6.831 & 30.062 & 0.3806 \\
\hline & PROM & 20.673 & 19.148 & 13.007 & 103.479 & 84.217 & 33.203 \\
\hline & MAX & 42.101 & 30.291 & 29.546 & 165.954 & 195.011 & 100.269 \\
\hline & $\mathrm{DE}$ & 0.9724 & 0.6667 & 0.7418 & 31.626 & 44.758 & 34.053 \\
\hline \multirow{4}{*}{ Valle de Azapa } & MIN & 0.5689 & 12.607 & 0.4636 & 79.227 & 32.809 & 0.6417 \\
\hline & PROM & 32.011 & 23.391 & 21.048 & 113.394 & 149.679 & 35.346 \\
\hline & MAX & 80.631 & 81.895 & 64.087 & 378.796 & 426.308 & 82.198 \\
\hline & $\mathrm{DE}$ & 19.121 & 18.027 & 15.507 & 80.695 & 101.484 & 20.289 \\
\hline \multirow{4}{*}{ Total } & $\mathrm{MIN}$ & 0.5223 & 10.662 & 0.2591 & 6.831 & 30.062 & 0.3806 \\
\hline & PROM & 26.342 & 2.127 & 17.027 & 108.436 & 116.948 & 34.274 \\
\hline & MAX & 80.631 & 81.896 & 64.087 & 378.796 & 426.308 & 100.269 \\
\hline & $\mathrm{DE}$ & 15.947 & 13.491 & 12.596 & 6.026 & 83.781 & 27.485 \\
\hline
\end{tabular}

* $\mu \mathrm{g} / \mathrm{g}$ de peso seco

MIN: valor mínimo, PROM: promedio, MAX: valor máximo, DE: desviación estándar

de contaminación ambiental (Frantz et al. 2012, Cai y Calisi 2016), lo que ha permitido confirmar daños ecosistémicos en regiones donde existen altos niveles de contaminación. Un elemento que favorece su utilidad en los análisis ecotoxicológicos es que sus poblaciones son numerosas y presentan amplios rangos de distribución, por lo que no hay problemas de conservación.

Este trabajo es uno de los primeros en evaluar la presencia y concentraciones de $\mathrm{Pb}, \mathrm{As}$ y $\mathrm{Cd}$ en poblaciones naturales del extremo norte de Chile. Previamente se han evaluado aves costeras de la ciudad de Arica (Maulén 2014), especies en que las concentraciones de $\mathrm{Pb}$ son consideradas bajas (0.15 $\pm 0.14 \mu \mathrm{g} / \mathrm{g}$ en hígado [0.00 a $0.49 \mu \mathrm{g} / \mathrm{g}$ ] y $0.26 \pm$ $0.24 \mu \mathrm{g} / \mathrm{g}$ en hueso [rango $0.07 \mathrm{a} 1.2 \mu \mathrm{g} / \mathrm{g}$ ]), particularmente porque se alimentan en amplias zonas geográficas, desplazándose incluso mar adentro, lugares poco relevantes con relación a la contaminación por metales pesados.

Las concentraciones promedio de $\mathrm{Pb}$ encontradas en palomas del radio urbano de la ciudad de Arica $(1.7 \mu \mathrm{g} / \mathrm{g}$ en tejido hepático y $10.84 \mu \mathrm{g} / \mathrm{g}$ en tejido óseo) sobrepasan las encontradas en aves costeras de la misma ciudad $(0.15 \mu \mathrm{g} / \mathrm{g}$ en tejido hepático y $0.26 \mu \mathrm{g} / \mathrm{g}$ en tejido óseo), y superan sustancialmente el máximo permitido en alimentos relacionados con tejidos biológicos, que es de 1 a $2 \mu \mathrm{g} / \mathrm{g}$ (MINSAL 1996). Al comparar tejidos óseos con hepáticos en las palomas, se observan diferencias significativas $(Z=-6.186, p=0.000)$; es decir, el hueso tiene entre seis a ocho veces más $\mathrm{Pb}$ que el hígado. A partir de esto se puede inferir que la contaminación a la que están expuestas las palomas ha sido permanente en el tiempo (Valladares et al. 2013). Sin embargo, como se observa en el cuadro II, hay lugares en el mundo donde se han encontrado concentraciones mucho más altas de este metal en hueso de palomas, como España (Antonio-García et al. 1988) e Inglaterra (Hutton y Goodman 1980), así como en China en otros tejidos, como pulmón (Cui et al. 2016, 2018).

Una alta exposición a $\mathrm{Cd}$ causa una significativa acumulación de este metal en tejidos blandos; a pesar de que una pequeña parte es excretada, permanece principalmente retenido en hígado y riñón (Webb y Caín 1982, Torra et al. 1994). Cuando el Cd alcanza la sangre, este elemento químico es retenido en el hígado, donde se estimula la síntesis de metalotioneinas, formando un complejo Cd-metalotioneina (Cd-MT) que es directamente transportado al riñón para su excreción (García-Rico et al. 1999). Sin embargo, la alta bioacumulación de Cd-MT en el riñón causa una gama de nefropatías, ya que afecta los túbulos proximales (Torra et al. 1994). En ese sentido, concentraciones sobre $6 \mu \mathrm{g} / \mathrm{g}$ en el riñón pueden causar bloqueo en la absorción de $\mathrm{Zn}$ y $\mathrm{Cu}$, originando varias nefropatías (Onosaka et al. 1984, Gallant y Cherian 1987, Ohta et al. 1989, Kaji et al. 1995, López-Alonso et al. 2012). Con relación a las concentraciones encontradas en palomas, también se consideran altas, ya que el promedio en todos los ejemplares analizados es de $11.7 \mu \mathrm{g} / \mathrm{g}$. Cabe destacar que, en todos los ejemplares analizados, las concentraciones de $\mathrm{Cd}$ en hígado fueron tres y siete veces 


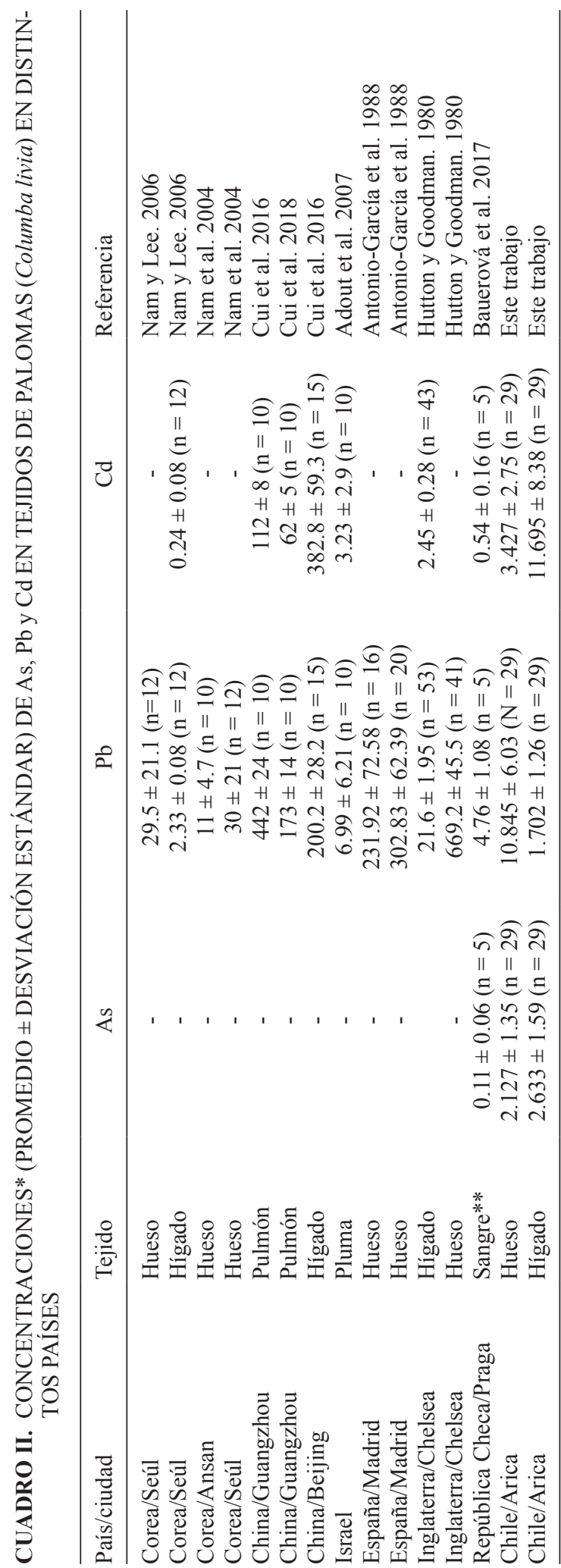

mayores que en hueso $(Z=-4.795, p=0.000)$, lo que tiene sentido tomando en cuenta que se bioacumula en tejidos blandos.

Por otro lado, el As es considerado un metaloide ampliamente distribuido en los ambientes naturales. Alrededor de 200 millones de personas se ven afectadas por el consumo de agua contaminada con As inorgánico que excede el límite máximo recomendado por la OMS $(10 \mu \mathrm{g} / \mathrm{L}$; Flanagan et al. 2012). Una serie de alimentos son ingeridos con altas concentraciones de este metaloide, como algas (Almela et al. 2002) y arroz, generando altas tasas de arsenicismo en las poblaciones (Díaz et al. 2015). El As ha sido clasificado como una de las 20 sustancias más peligrosas presentes en el ambiente (Hughes et al. 2011), siendo su forma inorgánica una de las más peligrosas, ya que se le considera carcinogénica para varios tejidos como hígado, riñón, vejiga, piel, sistema nervioso central y sistema digestivo, en el cual genera diarrea y gastroenteritis. De acuerdo con el análisis realizado por instituciones gubernamentales, se han cuantificado en el aire de la ciudad de Arica en promedio $31 \mathrm{~g} /$ $\mathrm{m}^{3}$ de partículas $\mathrm{PM}_{10}$ de As, y hasta $94.3 \mathrm{~g} / \mathrm{kg}$ en polvo precipitado dentro de las casas. Esto indica que los valores encontrados en palomas $(2.63 \mu \mathrm{g} / \mathrm{g})$ pueden considerarse más altas, más aún cuando el máximo permitido en alimentos relacionados con tejidos biológicos es de 1 a $2 \mu \mathrm{g} / \mathrm{g}$ (MINSAL 1996). Por otro lado, no existen diferencias significativas entre tejidos $(Z=-1.501, p=0.133)$; sin embargo, lo más relevante es que hay pocos estudios que hayan evaluado la presencia de As en palomas como bioindicadoras de contaminación, y hay escasa información de este metaloide en otras poblaciones de esta especie (Cuadro II)

\section{CONCLUSIONES}

A la luz de las concentraciones encontradas en palomas, es posible afirmar que aún después del plan de descontaminación de Arica, que se llevó a cabo hace ya varios años, se observan altos niveles de polimetales en especies que habitan en el radio urbano de la ciudad; por lo tanto, es importante seguir considerando medidas de mitigación que aceleren el proceso de disminución de estos elementos. Por otro lado, se estima que las palomas pueden ser bioindicadores importantes de contaminación ambiental, por lo que se plantea la necesidad de desarrollar un programa de monitoreo basado en la presencia y concentración de polimetales en 
palomas. Sin embargo, es necesario incluir otros elementos químicos en el análisis, como zinc, cobre y mercurio, así como otros tejidos como plumas, ya que éstas son el tejido más externo y el primero en entrar en contacto con contaminantes (Abdullah et al. 2015), y pulmones, que son los primeros órganos en recibir las partículas contaminantes respirables (Cui et al. 2016).

\section{AGRADECIMIENTOS}

Trabajo financiado por la Universidad de Tarapacá, proyecto de investigación para estudiantes de pregrado 2017, código 4719/17, "Determinación de plomo en tejidos biológicos de vertebrados presentes en áreas urbanas de la ciudad de Arica (Chile)", otorgado a la alumna Grimanesa Cáceres Tapia. Nuestros agradecimientos a Sue Ellen Vega y a todo el equipo de LASPAL por su apoyo durante la etapa de análisis químico de las muestras.

\section{REFERENCIAS}

Almela C., Algora S., Benito V., Clemente M.J., Devesa V., Súñer M.A., Vélez D. y Montoro R. (2002). Heavy metal, total arsenic, and inorganic arsenic contents in algae food products. J. Agric. Food Chem. 50(4), 918923. DOI: 10.1021/jf0110250

Close B., Banister K., Baumans V., Bernoth EM., Bromage N., Bunyan J., Erhardt W., Flecknell P., Gregory N., Hackbarth H., Morton D. y Warwick C. (1997). Recommendations for euthanasia of experimental animals: part 2. DGXT of the European Commission. Lab. Anim. 31: 1-32. DOI: 10.1258/002367797780600297

Cui J., Halbrook R.S., Zang S. y You Y. (2016). Use of homing pigeons as biomonitors of atmospheric metal concentrations in Beijing and Guangzhou, China. Ecotoxicology $25,439-446$.

DOI: $10.1007 / \mathrm{s} 10646-015-1601-\mathrm{y}$

Cui J., Halbrook R.S., Zang S., Han S. y Li X. (2018) Metal concentrations in homing pigeon lung tissue as a biomonitor of atmospheric pollution. Ecotoxicology 27, 169-174.

DOI 10.1007/s10646-017-1882-4
Díaz O.P., Arcos R., Tapia Y., Pastene R., Velez D., Devesa V., Montoro R., Aguilera V. y Becerra M. (2015). Estimation of arsenic intake from drinking water and food (raw and cooked) in a rural village of northern Chile. Urine as a biomarker of recent exposure. Int. J. Environ. Res. Public Health 12(5), 5614-5633. DOI: $10.3390 /$ ijerph 120505614.

Gallant K.R. y Cherian M.G. (1987). Changes in dietary zinc result in specific alteration pf metallothionein concentration in newborn rat liver. J. Nutr. 117, 708. DOI: $10.1093 / \mathrm{jn} / 117.4 .709$

García-Rico L. y Jara-Marini M. (1996). Aplicación de microondas en la digestión de hígado de bovino para la cuantificación de metales pesados. Rev. Int. Cont. Amb. 12, 40-44.

González-Acuña D., Silva F., Moreno L., Cerda F., Donoso S., Cabello J. y López J. (2007). Detección de algunos agentes zoonóticos en la paloma doméstica (Columba livia) en la ciudad de Chillán, Chile. Rev. Chil. Infectol. 24(3): 199-203.

DOI: $10.4067 /$ S0716-10182007000300004.

Kaji T., Mishima A., Machida M., Yabusaki K., Suzuki M., Yamamoto C., Fujiwara Y., Skamoto M. y Kosuka H. (1995). B. Environ. Contam. Tox. 54, 501-506. DOI: 10.1007/bf00192591

López-Alonso M., García-Vaquero M., Benedito J., Castillo C. y Miranda M. (2012). Trace mineral status and toxic metal accumulation in extensive and intensive pigs in NW Spain. Livestock Science 146(1), 47-53. DOI: 10.1016/j.livsci.2012.02.019

Ohta H., Deangeli Y. y Cherian M.G. (1989). Uptake of Cadmium and metallothionein by rat everted intestinal sac. Toxicol. Appl. Pharm. 101, 62-69.

DOI: $10.1016 / 0041-008 \times(89) 90212-3$

Onosaka S., Tanaka K. y Cherian M.G. (1984). Effects of cadmium and zinc on tissue levels of metallothionein. Environ. Health Perspect. 54, 67-72.

DOI: $10.1289 /$ ehp. 845467

Torra M., Figueras J., Brunet M., Rodamilans M. y Corbella J. (1994). Total and metallothionein-bound cadmium in the liver and kidney of a population in Barcelona (Spain). B. Environ. Contam. Tox. 53, 509515. DOI: $10.1007 / \mathrm{BF} 00199019$

Webb M. y Cain K. (1982). Functions of metallothionein. Biochem. Pharmacol. 31(2), 137-142. DOI: $10.1016 / 0006-2952(82) 90202-7$ 